\title{
Optical microvariability of bright type 2 quasars
}

\author{
Jana Polednikova ${ }^{1,2}$, Alessandro Ederoclite ${ }^{3}$, Jordi Cepa ${ }^{1,2}$, \\ José Antonio de Diego Onsurbe ${ }^{4}$ and José Ignacio González-Serrano ${ }^{5}$ \\ ${ }^{1}$ Instituto de Astrofísica de Canarias, \\ La Laguna, Spain \\ email: jana@iac.es \\ ${ }^{2}$ Universidad de La Laguna, La Laguna, Spain \\ ${ }^{3}$ Centro de Estudios de Física del Cosmos de Aragón, Teruel, Spain \\ ${ }^{4}$ Instituto de Astronomía, Universidad Autónoma de México, \\ México D. F., México \\ ${ }^{5}$ Instituto de Física de Cantabria, CSIC-Universidad de Cantabria, \\ Santander, Spain
}

\begin{abstract}
We present results from a project focused on searching optical microvariabilty (also known as "intra-night" variability) in type 2 - obscured - quasars. Optical microvariability can be described as very small changes in the flux, typically in the order of hundredths of magnitude, which can be observed on timescales of hours. Such studies have been so far conducted for samples of blazars and type 1, unobscured, AGNs, where the optical microvariability was detected with success. We have focused on obscured targets which would pose a challenge to the AGN standard model. In the present work, however, we have observed a sample of three bright ( $\mathrm{g}$ mag $<17)$ type 2 quasar, based on the catalog of type 2 quasars from SDSS of Reyes et al. $(2008)$. The observations were carried out with the 1.5 meter telescope at San Pedro Martir observatory in Mexico. The sample was observed during an observation period of four days in Johnsons $\mathrm{V}$ filter, resulting in at least two continuous intervals of observations per target during the observational run. We have obtained differential light curves for our sources as well as for the comparison stars. They were analyzed using one-way analysis of variance statistical test (ANOVA), which has been repeatedly used in the past for studies of unobscured targets. Based on the results from the statistical analysis, we show that at least two out of three observed targets appear to be variable on time scales of hours. So far, this is the first study which confirmed existence of optical microvariability in type 2 quasars.
\end{abstract}

Keywords. galaxies: active, quasars: individual (Mrk 477, SDSS J075+5050, SDSS J1430+1339)

\section{Introduction}

The standard model for active galactic nuclei (AGNs) introduced by Antonucci (1993) assumes that the cause of existence of different classes of AGNs is due to the diverse orientation of the obscuring torus. If the obscuring torus is blocking our line of sight and therefore the direct view onto the central engine of the AGN, we are talking about optically obscured targets (type 2) while in the case of higher inclination angles we consider the targets to be optically unobscured (type 1).

Variability is a crucial characteristics of AGNs. It is well studied (in many cases) at all wavelengths and at various time scales. It is a powerful tool to probe the physical mechanisms in the otherwise inaccessible parts of the system.

Microvariability (or intra - night variability) can be defined as a flux changes on short time scales, from minutes to hours, with magnitudes ranging from hundredths to tenths 
Table 1. Properties of the observed targets from the Reyes et al. (2008) catalog. Right ascension and declination are in J2000.0. L[OIII] is in solar luminosities. Brightness in V filter was transformed from SDSS filters, values correspond to A catalogue of quasars and active nuclei: 13th edition (Véron-Cetty \& Véron (2010)).

\begin{tabular}{cccccc}
\hline \hline Target & R. A. & dec & $V_{\operatorname{mag}}$ & $z$ & $L_{[\mathrm{OIII}]}$ \\
\hline J0759+5050 & $07: 59: 40.96$ & $+50: 50: 24.89$ & 16.59 & 0.055 & $10^{8.77}$ \\
J1430+1339 & $14: 30: 29.88$ & $+13: 39: 12.09$ & 17.64 & 0.085 & $10^{9.04}$ \\
Mrk 477 & $14: 40: 38.09$ & $+53: 30: 19.9$ & 15.03 & 0.037 & $10^{8.72}$ \\
\hline
\end{tabular}

of magnitude. While the first reports of microvariability can be found in Matthews \& Sandage (1963), the studies became reliable only after introducing more reliable CCD detectors. Nevertheless microvariability was mostly studied in the case of blazars (Carini 1990) and unobscured type 1 AGNs (Ramírez et al. 2004), since the study of such phenomena in optically obscured targets is challenging due to their intrinsic low brightness and high contribution from the host galaxy, which is essentially blocking our view of the central engine. Following the causality, rapid variations should originate in the innermost parts of the AGN, which makes microvariability a perfect tool to study the morphology of the central part of the AGN.

We are presenting results from the pilot study focused on microvariability of type 2 quasars. We report detection of microvariability in two out of three cases, which might pose a challenge to the unified scenario.

\section{Sample and observations}

We have selected the brightest targets (Vmag < 17) from the Reyes et al. (2008) catalog, based on SDSS DR6 spectroscopic database (Adelman-McCarthy et al. 2008). There are four targets fulfillingthe observational requirementa, but only three of them were observed due to the technical difficulties of the telescope. The observations were carried out with the 1.5 meter telescope at the San Pedro Martír observatory at Baja California, México during four nights in March 2011. The majority of the observations were taken in Johnson's V filter, with control color observations taken in Johnson's R and B. Exposure time was set to 60 seconds, which is sufficient to obtain signal to noise $>100$. Such high $\mathrm{S} / \mathrm{N}$ allows us to look for variations of order 0.01 mag. Each target was observed for more than two hours per night, which, including overheads, yields more than 100 data points per light curve. The sample is summarized in Table 1.

\section{Data analysis}

The data were reduced using the standard tasks from the IRAF data reduction software. Cosmic ray removal was performed using LAcos (van Dokkum 2001). Aperture photometry was carried out using SExtractor (Bertin \& Arnouts 1996). We assume that the host galaxy contribution is constant, hence including all the light from the galaxy into the aperture, doesn't affect the variability study (Cellone et al. 2000). We benefited from presence of different stars in the field, hence we used differential photometry.

The analysis itself was carried out using one way analysis of - ANOVA. Which proves to be a robust test for detecting variations as proved by de Diego (2010). We have obtained two light curves, one is a constant curve of difference between comparison and reference star, while the other light curve is an actual target - reference star. For each light curve, we have divided the data into sets of five data points. Within each set, the 

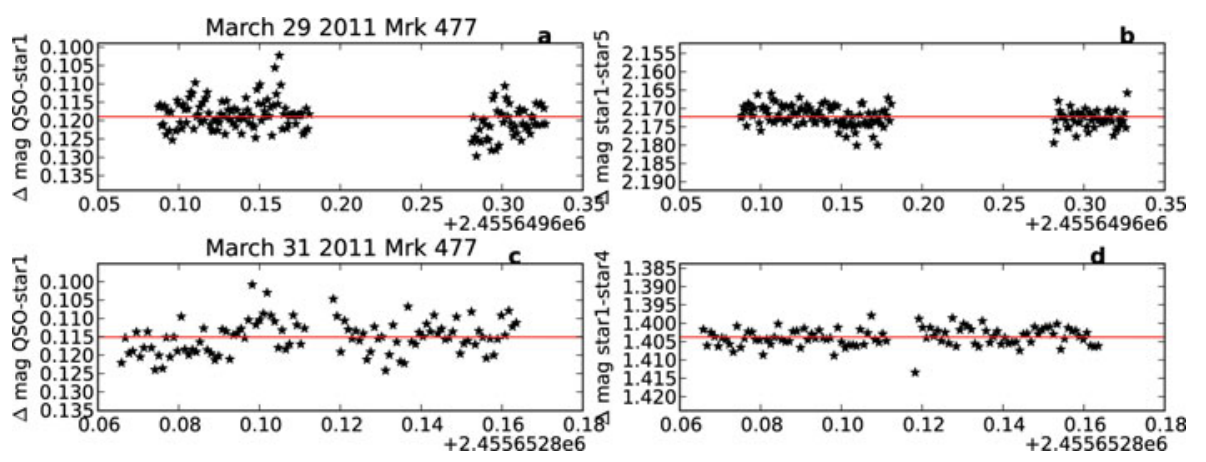

Figure 1. Differential light curves for Mrk 477 in $\mathrm{V}$ filter. Left panels are representing $m_{Q S O}-m_{c o m p}$, right ones are $m_{c o m p}-m_{c h e c k}$. Each line represents one observing night. Red line represents mean values. Outlying points were not used for the ANOVA analysis.

mean and the variances are computed. These sets are then used to compute the variance of the whole sample. For further mathematical explanation of ANOVA see de Diego et al. (1998). The outcome is $\alpha$ - level of significance. We consider the target to be variable if the level of significance, $\alpha \leqslant 0.001$, while targets with level of significance in the range $0.1 \leqslant \alpha \leqslant 0.95$ are considered to be constant. In this case, extremes are unlikely to happen and if so is the case, they need careful examination for underlying trends etc. In the case where $0.001 \leqslant \alpha \leqslant 0.1$, the results are considered ambiguous and we refrain from drawing any conclusions from the data.

\section{Results and conclusions}

We have carried ANOVA analysis for three type 2 quasars. We got a positive result of microvariability in both observing nights for Mrk 477 and in one night (out of three) for J0759+5050. In the case of J0759+5050 we have observed a change of 0.4 mag. Mrk 477 shows variability of 0.027 mag on March 292011 and 0.023 mag on March 312011. Mrk 477 seem to be one of the thoughtfully observed targets, since it was included also in the spectropolarimetric study by Tran et al. (2000), which confirmed existence of the hidden broad line region. Jang (2001) already reported some microvariability in Mrk 477, however it lacks details.

According to the unified model, we should be unable to detect variation of any sort, since our view onto the innermost parts of the AGN is blocked by the disty torus. Nevertheless long term variations have been already detected in a few Seyfert 2 galaxies, as well as short term x-ray variability (Awaki et al. 2006). Yet optical short term variability was up till know an undetected phenomena in type 2 quasars.

One of the plausible explanation for detecting microvariations can be misclassification of the targets. However to further explore this possibility, we would need to obtain more precise spectra than provided by present large scale surveys like SDSS. Another option is view onto the central engine through the structure in the dusty torus. While the original unified scenario introduced by Antonucci (1993) assumed homogeneous dusty torus surrounding the central part of the AGN, Krolik \& Begelman (1988) suggests that such structure would be hard to maintain and hence the torus should consists of clumps. If there is a 'window' between the clumps, it might provide an opportunity to see the central part of the AGN. However a model proposed by Nenkova et al. (2008) assumes only six or seven such clumps, which seem unlikely explanation to the microvariations reported here. Another explanation might involve the polarized light. Charged particles 
which are forming a corona on top of the accretion disc are known to polarize light from the central engine. Such polarized light carries informations about the central region. As mentioned above, we can detect the broad lines as reflected by the polarized light. We assume that the reflected light might vary according to the variations originating in the central engine. We know that Mrk 477 harbors hidden broad line (Tran et al. 2000), therefore it might be a plausible explanation. In the case of another two targets, we don't have any information about the polarized spectra, hence we cannot draw conclusion in those cases.

An alternative explanation might lie in the X-ray observations. Type 2 targets exhibit rapid variations in the X-ray continuum, higher than can be attributed solely to the central engine. A solution was proposed by Risaliti et al. (2005a) (and references therein). According to them, the broad line region harbors clouds with different neutral hydrogen absorption column, which are passing through our line of sight. The proposed alternative suggests that the changes in the line of sight, produced by such clouds can propagate further into the medium and cause microvariations. However no long x-ray observations of our sample were carried out so far.

\section{Acknowledgment}

This research has been supported by the Spanish Ministerio de Economía y Competitividad (MINECO) under the grant AYA2011-29517-C03-01. J. A. de Diego is grateful for support from grants SAB2010-0011 awarded by the Spanish MIED, and PAPIIT IN110013 awarded by the UNAM. J. I. González-Serrano is grateful for the support from AYA2011-29517-C03-02. The authors also would like to thank our colleague Yair Krongold for useful suggestions on this paper.

\section{References}

Adelman-McCarthy, J. K. et al. 2008, APJS, 175, 297

Antonucci, R. R. J. 1993, ARAA, 31, 473

Awaki, H., Murakami, H., Ogawa, Y., \& Leighly, K. M. 2006, APJ, 645, 928

Bertin, E. \& Arnouts, S. 1996, AAP, 117, 393

Carini, M. T. 1990, BAAS, 22R1337C

Cellone, S. A., Romero, G. E., \& Combi, J. A. 2000, AJ, 119, 1534

de Diego, J. A., Dultzin-Hacyan, D., Ramírez, A., \& Benítez, E. 1998, APJ, 500, 69

de Diego, J. A. 2010, $A J, 139,1269$

van Dokkum, P. G. 2001, PASP, 113, 1420

Jang, M. 2001 APSS, 275, 209

Krolik, J. \& Begelman, M. C. 1988, APJ, 329, 702

Matthews, T. A. \& Sandage, A. R. 1963, APJ, 138, 30

Nenkova, M., Sirocky, M. M., Ivezić, Ž., \& Elitzur, M. 2008 APJ, 685, 147

Ramírez, A., de Diego, J. A., Dultzin, D., \& González-Peréz, J.-N. 2004, A\&\&A, 421, 83

Reyes, R., Zakamska, N. L., Strauss, M. A., Green, J., Krolik, J. H., Shen, Y., Richards, G. T., Anderson, S. F., \& Schneider, D. P. 2008, AJ, 136, 2373

Risaliti, G., Elvis, M., Fabbiano, G., Baldi, A., \& Zezas, A. 2005, APJ, 623, 93

Tran, H. D., Cohen, M. H., \& Villar-Martin, M. 2000 AJ, 120, 562

Véron-Cetty, M.-P., \& Véron, P. 2010 A\& $A, 518,10$ 